\title{
Stress intensity factors around a moving Griffith crack in an infinite elastic layer between two elastic half-planes
}

\author{
Shouetsu ITOU \\ Department of Mechanical Engineering, Kanagawa University, \\ Rokkakubashi, Kanagawa-ku, Yokohama 221-8686, Japan \\ E-mail : itous001@kanagawa-u.ac.jp
}

\begin{abstract}
Stresses around a propagating finite crack having a constant velocity in an elastic layer sandwiched between two elastic half-planes are determined. The self-equilibrated system of pressure is applied to the crack surfaces. Application of the Fourier transform technique reduces the problem to that of solving dual integral equations. In order to solve the equations, the differences of the crack surface displacements are expanded in a series of functions that are equal to zero outside of the crack. The unknown coefficients in the series are solved using the Schmidt method. The stress intensity factors are calculated numerically for a crack in a layer made of epoxy resin sandwiched between two half-planes made of aluminum.
\end{abstract}

Key Word : Moving crack, Yoffe model, Stress intensity factor, Composite materials, Fourier transform technique, Schmidt method

\section{Introduction}

Fiber reinforced plastics (FRP) have been widely used as structural members in airplanes, automobiles and high-speed trains because these materials offer sufficient strength and are lightweight. Consider the case in which a matrix made of epoxy resin is reinforced by aluminum fiber. In the matrix, defects such as voids or inclusions are inevitably present. Under the application of a load, high stresses occur around these defects, and finally a crack may appear in the epoxy material. Static stresses in composite materials that have been weakened by a crack have been solved in detail by Erdogan and Gupta [1]. Sih and Chen determined the transient dynamic stress field around a crack in an infinite layer sandwiched between two elastic half-planes [2]. Later, they considered the Yoffe model [3] for a moving crack in layered composites [4]. In the Yoffe model, the crack propagates to the right only and maintains a constant length. Therefore, this model is not substantial. As the propagating velocity of a crack increases, the crack divides into two branches. Yoffe revealed that the maximum circumferential stress shifts out of the plane of the crack when the propagation velocity exceeds a certain value and clarified well the characteristics of the crack branching.

Sih and Chen [4] situated a moving crack on a mid-plane of an infinite layer sandwiched between two elastic half-planes, and determined the stress field in the composite materials that have symmetry with respect to the crack. In contrast, Chen, Keer and Achenbach considered that a crack of the Yoffe model propagates parallel to the interface of the two dissimilar elastic half-planes [5]. To the author's knowledge, an unsymmetric problem with respect to a moving crack has not been investigated for the layered materials.
In the present paper, the Yoffe model has been applied for a moving Griffith crack in an infinite elastic layer sandwiched by two elastic half-planes. The crack is not placed on a mid-plane of the layer, and the boundary conditions of the problem are not symmetric with respect to the crack. A self-equilibrating normal traction is applied to the surfaces of the crack. Application of the Fourier transform technique reduces the problem to that of solving a pair of dual integral equations. In order to solve the equations, the differences of the crack surface displacements are expanded in a series of functions that are zero outside the crack. The unknown coefficients in the series are solved using the Schmidt method [6].

Although the stress intensity factors $K_{1}$ and $K_{2}$ can be easily determined even for a moving crack in an elastic layer, the present author was previously unable to obtain the circumferential stress intensity factor $K_{\theta \theta}$ around the crack end. (The reason for this is described in detail in Section 5.) Recently, however, the author was able to determine $K_{\theta \theta}$ around the crack end.

Numerical calculations are carried out for the circumferential stress intensity factor $K_{\theta \theta}$ for the case in which a layer made of epoxy resin is sandwiched by two half-planes made of aluminum.

\section{Fundamental Equations}

With reference to the fixed rectangular coordinate system, the equations of motion in the plane state of strain are reduced to the forms

$$
\begin{aligned}
& \partial^{2} \phi^{*} / \partial x^{* 2}+\partial^{2} \phi^{*} / \partial y^{* 2}=1 / c_{L}^{2} \times \partial^{2} \phi^{*} / \partial t^{* 2} \\
& \partial^{2} \phi^{*} / \partial x^{* 2}+\partial^{2} \phi^{*} / \partial y^{* 2}=1 / c_{L}^{2} \times \partial^{2} \phi^{*} / \partial t^{* 2}
\end{aligned}
$$

with

$$
u^{*}=\partial \psi^{*} / \partial x^{*}-\partial \psi^{*} / \partial y^{*}, \quad v^{*}=\partial \psi^{*} / \partial x^{*}+\partial \psi^{*} / \partial y^{*}
$$




$$
c_{L}^{2}=(\lambda+\mu) / \rho, \quad c_{T}^{2}=\mu / \rho
$$

where displacements $u^{*}$ and $v^{*}$ are defined as $x^{*}$ and $y^{*}$ components, respectively, and $c_{L}$ and $c_{T}$ are the longitudinal and transversal elastic wave velocities, respectively. In Eq. (3), $\lambda$ and $\mu$ are the Lame constants and $\rho$ is the density of the material.

Consider an infinite layer sandwiched between upper half-plane (3) and lower half-plane (4), as shown in Fig. 1. A cracked layer is divided into layer (1) denoted by $0 \leq y^{*} \leq h_{1}$ and layer (2) denoted by $-h_{2} \leq y^{*} \leq 0$. The crack is assumed to be opened at one end and closed at the other, has constant speed $U$ and maintains its length to be $2 a$ in the layer. For a moving crack with a constant speed, it is convenient to introduce the Galilean transformation

$$
x=x^{*}-U t^{*}, \quad y=y^{*}, \quad t=t^{*}
$$

where $(x, y)$ is the translating coordinate system attached to the moving crack. In the moving coordinates, Eq. ( 1 ) becomes independent of the time variable and can be converted to the forms

$$
\begin{aligned}
& \partial^{2} \phi / \partial x^{2}+\partial^{2} \phi / \partial y^{2}=M_{L}^{2} \partial^{2} \phi / \partial x^{2}, \\
& \partial^{2} \phi / \partial x^{2}+\partial^{2} \phi / \partial y^{2}=M_{L}^{2} \partial^{2} \phi / \partial x^{2}
\end{aligned}
$$

with

$$
M_{T}=U / c_{T}, \quad M_{L}=U / c_{L}=\{(1-2 v) /[2(1-v)]\}^{1 / 2} \times M_{T}
$$

where $M_{T}$ and $M_{L}$ represent the Mach numbers of the moving crack with respect to the longitudinal and transversal elastic wave velocities, respectively, and $v$ is the Poisson's ratio. The displacements and the stresses are expressed by the equations

$$
\begin{aligned}
& u=\partial \phi / \partial x-\partial \phi / \partial y, \quad v=\partial \phi / \partial x+\partial \phi / \partial y \\
& \tau_{y y} / \mu=\left(M_{T}^{2}-2\right) \partial^{2} \phi / \partial x^{2}+2 \partial^{2} \phi / \partial x \partial y \\
& \tau_{x x} / \mu=\left(2+M_{T}^{2}+2 M_{L}^{2}\right) \partial^{2} \phi / \partial x^{2}-2 \partial^{2} \phi / \partial x \partial y \\
& \tau_{x y} / \mu=2 \partial^{2} \phi / \partial x \partial y-\left(M_{T}^{2}-2\right) \partial^{2} \phi / \partial x^{2} .
\end{aligned}
$$

\section{Boundary Conditions}

If the applied tensile load acts on the composite materials perpendicular to the layer, the boundary conditions for the problem can be expressed as follows :

$$
\begin{array}{ll}
\tau_{y y 1}=\tau_{y y 3}, \quad \tau_{x y 1}=\tau_{x y 3}, & u_{1}=u_{3}, \quad v_{1}=v_{3}, \text { at } y=h_{1},|x| \leq \infty \\
\tau_{y y 1}=\tau_{y y 2}, \quad \tau_{x y 1}=\tau_{x y 2} & \text { at } \quad y=0,|x| \leq \infty \\
\tau_{y y 1}=-p, \quad \tau_{x y 1}=0 & \text { at } \quad y=0,|x| \leq \infty \\
u_{1}=u_{2}, \quad v_{1}=v_{2} & \text { at } \quad y=0, \quad a \leq|x|
\end{array}
$$

$$
\tau_{y y 2}=\tau_{y y 4}, \quad \tau_{x y 2}=\tau_{x y 4}, u_{2}=u_{4}, \quad v_{2}=v_{4} \text { at } y=-h_{2},|x| \leq \infty
$$

where $p$ is a constant, and subscript indicates layers $i(i=1,2)$ and half-planes $i(i=3,4)$.

\section{Analysis}

We use the Fourier transforms defined by the equations

$$
\begin{aligned}
& \bar{f}(\xi)=\int_{-\infty}^{\infty} f(x) \exp (i \xi x) d x, \\
& f(x)=1 /(2 \pi i) \times \int_{-\infty}^{\infty} \bar{f}(\xi) \exp (-i \xi x) d \xi .
\end{aligned}
$$

Applying Eq. (13.1) to Eq. (5) results in

$$
\left[d^{2} / d y^{2}-\left(1-M_{L}^{2}\right) \xi^{2}\right] \bar{\phi}=0, \quad\left[d^{2} / d y^{2}-\left(1-M_{T}^{2}\right) \xi^{2}\right] \bar{\phi}=0
$$

The solutions of Eq. (14) are in the following forms for layers $i(i=1,2)$, upper half-plane (3) and lower half-plane (4), respectively:

$$
\begin{aligned}
& \bar{\phi}_{i}=A_{i} \cosh \left(\alpha_{i} \xi y\right)+B_{i} \sinh \left(\alpha_{i} \xi y\right), \\
& \bar{\phi}_{i}=C_{i} \cosh \left(\beta_{i} \xi y\right)+D_{i} \sinh \left(\beta_{i} \xi y\right), \\
& \bar{\phi}_{3}=A_{3} \exp \left(-\alpha_{3}|\xi| y\right), \quad \phi_{3}=C_{3} \exp \left(-\beta_{3}|\xi| y\right) \\
& \bar{\phi}_{4}=A_{4} \exp \left(\alpha_{4}|\xi| y\right), \quad \phi_{4}=C_{4} \exp \left(\beta_{4}|\xi| y\right)
\end{aligned}
$$

with

$$
\alpha_{i}=\left(1-M_{L i}^{2}\right)^{1 / 2}, \quad \beta_{i}=\left(1-M_{T i}^{2}\right)^{1 / 2}
$$

where the unknown coefficients $A_{1}, B_{1}, \sim, C_{4}$ are to be determined from the boundary conditions.

Next, we substitute Eqs. (15) (17) into the Fourier transformed expressions of Eqs. (7) and (8). The boundary conditions (9) and (12) are valid for the entire region of $x$, and these equations are easily satisfied in the Fourier transform domain. Then, the displacements $u_{1}, v_{1}, u_{3}, v_{3}$ and the stresses $\tau_{y y 1}, \tau_{x y 1}, \tau_{y y 3}, \tau_{x y 3}$ can be expressed by $C_{1}$ and $D_{1}$ only. Also, the displacements and stresses in layer (2) and those in half-plane (4) can be given by $C_{2}$ and $D_{2}$. For example, the displacements at $y=0$ are given by

$$
\begin{aligned}
& \bar{u}_{1}^{0}=C_{1} a_{11}^{(1)}+D_{1} a_{12}^{(1)}, \quad-i \bar{v}_{1}^{0}=C_{1} a_{21}^{(1)}+D_{1} a_{22}^{(1)} \\
& \bar{u}_{2}^{0}=C_{2} a_{11}^{(2)}+D_{2} a_{12}^{(2)}, \quad-i \bar{v}_{2}^{0}=C_{2} a_{21}^{(2)}+D_{2} a_{22}^{(2)}
\end{aligned}
$$

where the expressions of known functions $a_{11}^{(1)}, a_{12}^{(1)}, \cdots, a_{22}^{(2)}$ are shown in Appendix A, and variables having the superscript 0 are those at $y=0$. Using Eq. (19), $C_{1}$ and $D_{1}$ can be solved for $\tilde{u}_{1}^{0}$ and $-i \bar{v}_{1}^{0}$. In addition, $C_{2}$ and $D_{2}$ can be given by $\bar{u}_{2}^{0}$ and $-i \bar{v}_{2}^{0}$. Then, the stress components can be shown by the displacements at $y=0$. For example, $\bar{\tau}_{y y 1}^{0},=\bar{\tau}_{x y 1}^{0}, \bar{\tau}_{y y 2}^{0}$ and $\bar{\tau}_{x y 2}^{0}$ have the forms

$$
\begin{aligned}
& \bar{\tau}_{y y 1}^{0}=(-i) \bar{u}_{1}^{0} k_{1}^{(1)}(\xi)+\bar{v}_{1}^{0} k_{2}^{(1)}(\xi) \\
& \bar{\tau}_{x y 1}^{0}=i(-i) \bar{u}_{1}^{0} k_{3}^{(1)}(\xi)+i \bar{v}_{1}^{0} k_{4}^{(1)}(\xi)
\end{aligned}
$$




$$
\bar{\tau}_{x y 2}^{0}=i(-i) \bar{u}_{2}^{0} k_{3}^{(2)}(\xi)+i \bar{v}_{2}^{0} k_{4}^{(2)}(\xi)
$$

where the expressions of known functions $k_{1}^{(1)}(\xi), k_{2}^{(1)}(\xi), \cdots, k_{4}^{(2)}$ $(\xi)$ are shown in Appendix B.

Using Eq. (10), $\bar{u}_{1}^{0}$ and $\bar{v}_{1}^{0}$ can be expressed by $\bar{u}_{2}^{0}$ and $\bar{v}_{2}^{0}$ as follows :

$$
\begin{aligned}
(-i) \bar{u}_{1}^{0}=(-i) \bar{u}_{2}^{0} l_{1}(\xi)+\bar{v}_{2}^{0} l_{2}(\xi) \\
\bar{v}_{1}^{0}=(-i) \bar{u}_{2}^{0} l_{3}(\xi)+\bar{v}_{2}^{0} l_{4}(\xi)
\end{aligned}
$$

where the expressions of known functions $l_{1}(\xi), l_{2}(\xi), l_{3}(\xi)$ and $l_{4}(\xi)$ are shown in Appendix C.

In order to satisfy Eq. (11.2), the differences of the displacements at $y=0$ are expanded in the following series:

$$
\begin{aligned}
\left(v_{1}^{0}-v_{2}^{0}\right) & =\sum_{n=1}^{\infty} c_{n} \cos \left[(2 n-1) \sin ^{-1}(x / a)\right] & & \text { for }|x| \leq a \\
& =0 & & \text { for } \quad a \leq|x| \\
\left(u_{1}^{0}-u_{2}^{0}\right) & =\sum_{n=1}^{\infty} d_{n} \sin \left[2 n \times \sin ^{-1}(x / a)\right] & & \text { for } \quad|x| \leq a \\
& =0 & & \text { for } \quad a \leq|x|
\end{aligned}
$$

where $c_{n}$ and $d_{n}$ are unknown coefficients. The Fourier transformed expressions of Eqs. (24) and (25) are

$$
\begin{aligned}
& \left(\bar{v}_{1}^{0}-\bar{v}_{2}^{0}\right)=\sum_{n=1}^{\infty} c_{n}(2 n-1) / \xi \times J_{2 n-1}(a \xi) \\
& (-i)\left(u_{1}^{0}-u_{2}^{0}\right)=\sum_{n=1}^{\infty} d_{n} \times 2 n / \xi \times J_{2 n}(a \xi)
\end{aligned}
$$

where $J_{n}(\xi)$ is the Bessel functions. Using Eqs. (23) and (26), we can show the Fourier transformed displacements at $y=0$ by the coefficients $c_{n}$ and $d_{n}$. Then, the entire stress and displacement fields can be expressed by the coefficients. For example, $\bar{\tau}_{y y 2}^{0}$ and $\tau_{x y 2}^{0}$ can be represented by the forms :

$$
\begin{gathered}
\tau_{y y 2}^{0}=\sum_{n=1}^{\infty} c_{n}(2 n-1) / \pi \times \int_{0}^{\infty} Q_{1}(\xi) / \xi \times J_{2 n-1}(\xi a) \cos (\xi x) d \xi \\
+\sum_{n=1}^{\infty} d_{n}(2 n) / \pi \times \int_{0}^{\infty} Q_{2}(\xi) / \xi \times J_{2 n}(\xi a) \cos (\xi x) d \xi \\
\tau_{x y 2}^{0}=\sum_{n=1}^{\infty} c_{n}(2 n-1) / \pi \times \int_{0}^{\infty} Q_{3}(\xi) / \xi \times J_{2 n-1}(\xi a) \sin (\xi x) d \xi \\
+\sum_{n=1}^{\infty} d_{n}(2 n) / \pi \times \int_{0}^{\infty} Q_{4}(\xi) / \xi \times J_{2 n}(\xi a) \sin (\xi x) d \xi
\end{gathered}
$$

where the expressions of known functions $Q_{1}(\xi), Q_{2}(\xi), Q_{3}(\xi)$ and $Q_{4}(\xi)$ are shown in Appendix D. Functions $Q_{2}(\xi)$ and $Q_{3}(\xi)$ are identical and decrease rapidly as $\xi$ increases. Functions $Q_{1}(\xi)$ and $Q_{4}(\xi)$ have the following property when $\xi$ increases :

$$
Q_{i}(\xi) / \xi \rightarrow Q_{i}^{L} \quad(i=1,4)
$$

where constants $Q_{i}^{L}(i=1,4)$ can be calculated by

$$
Q_{i}^{L}=Q_{i}\left(\xi_{L}\right) / \xi_{L}
$$

where $\xi_{L}$ is a large value of $\xi$.

Finally, the remaining boundary condition (11.1) can be reduced to the equation

$$
\sum_{n=1}^{\infty} c_{n} G_{n}(x)+\sum_{n=1}^{\infty} d_{n} H_{n}(x)=-p
$$

$$
\sum_{n=1}^{\infty} c_{n} K_{n}(x)+\sum_{n=1}^{\infty} d_{n} L_{n}(x)=0 \quad \text { for } 0 \leq x \leq a
$$

where

$$
\begin{aligned}
& \left.\begin{array}{r}
G_{n}(x)=(2 n-1) / \pi \times\left\{\int_{0}^{\infty}\left[Q_{1}(\xi) / \xi-Q_{1}^{L}\right] J_{2 n-1}(a \xi) \cos (\xi x) d \xi\right. \\
+
\end{array} \quad Q_{1}^{L} \cos \left[(2 n-1) \sin ^{-1}(x / a)\right] /\left(a^{2}-x^{2}\right)^{1 / 2}\right\} \\
& H_{n}(x)=2 n / \pi \times \int_{0}^{\infty} Q_{2}(\xi) / \xi \times J_{2 n}(a \xi) \cos (\xi x) d \xi \\
& K_{n}(x)=(2 n-1) / \pi \times \int_{0}^{\infty} Q_{3}(\xi) / \xi \times J_{2 n-1}(a \xi) \sin (\xi x) d \xi \\
& L_{n}(x)=2 n / \pi \times\left\{\int _ { 0 } ^ { \infty } \left[Q_{4}(\xi) / \xi-Q_{4}^{L}(a \xi) \sin (\xi x) d \xi\right.\right. \\
& \left.+Q_{4}^{L} \sin \left[2 n \sin ^{-1}(x / a)\right] /\left(a^{2}-x^{2}\right)^{1 / 2}\right\} .
\end{aligned}
$$

The unknown coefficients $c_{n}$ and $d_{n}$ in Eq. (30) can now be solved by applying the Schmidt method [6].

\section{Circumferential Stress Intensity Factor}

In order to determine the circumferential stress intensity factor, the polar co-ordinate system $(r, \theta)$ is taken as shown in Fig. 2. The moving rectangular co-ordinates $(x, y)$ are related to the polar co-ordinates $(r, \theta)$ by the equation

$$
x=a+r \cos \theta, \quad y=r \sin \theta .
$$

The circumferential stress $\tau_{\theta \theta}$ is calculated by

$$
\tau_{\theta \theta}=\tau_{x x} \sin ^{2} \theta+\tau_{y y} \cos ^{2} \theta-2 \tau_{x y} \sin \theta \cos \theta .
$$

In fracture mechanics, when the maximum value of $K_{\theta \theta}$ at the crack tip exceeds the critical value known as the fracture toughness value of the material, the material is cleaved.

For a small value of $r$, the following relations are provided

$$
\begin{aligned}
& \int_{0}^{\infty} J_{n}(a \xi) \exp (-\beta y \xi) \exp (-i \xi) d \xi \\
& =(-i)^{n}\left\{\left[-\cos \theta+\left(\cos ^{2} \theta+\beta^{2} \sin ^{2} \theta\right)^{1 / 2}\right]^{1 / 2}\right. \\
& \left.-i\left[\cos \theta+\left(\cos ^{2} \theta+\beta^{2} \sin ^{2} \theta\right)^{1 / 2}\right]^{1 / 2}\right\} /\left[4 \operatorname{ar}\left(\cos ^{2} \theta+\beta^{2} \sin ^{2} \theta\right)^{1 / 2}\right] \\
& \text { for }(\beta y) \geq 0 .
\end{aligned}
$$

Equation (34) can be derived from the following formula [7]

$$
\begin{aligned}
& \int_{0}^{\infty} J_{n}(a \xi) \exp (-b \xi) d \xi \\
& =\left[\left(a^{2}+b^{2}\right)^{1 / 2}-b\right]^{n} /\left[a^{n}\left(a^{2}+b^{2}\right)\right]^{1 / 2} \\
& \qquad \text { for } n \geq 0, \operatorname{Re} a l(b \pm i a) \geq 0 .
\end{aligned}
$$

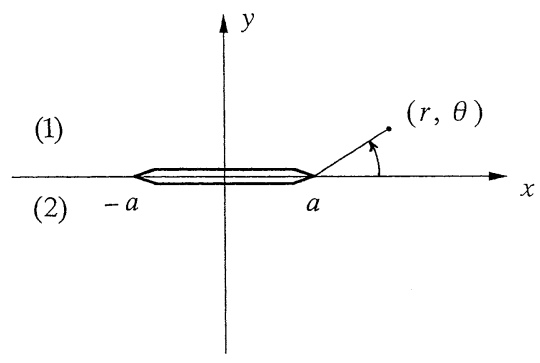

Figure 2 Polar coordinate system taken at the crack end. 
Ordinarily, the stress intensity factors can be defined using Eq.(34). Here, let us consider the stress $\tau_{y y 1}$ given by the form

$$
\begin{aligned}
\tau_{y y 1}^{0}=\sum_{n=1}^{\infty} c_{n}(2 n-1) / \pi \times \int_{0}^{\infty} & \left\{m_{1}(\xi) f_{1}(\xi)\left(M_{T 1}^{2}-2\right) \xi \cosh \left(\alpha_{1} \xi y\right)\right. \\
& +m_{1}(\xi) f_{2}(\xi)\left(M_{T 1}^{2}-2\right) \xi \sinh \left(\alpha_{1} \xi y\right) \\
& +m_{1}(\xi) \times 2 \beta_{1} \xi \sinh \left(\beta_{1} \xi y\right) \\
& -m_{3}(\xi) g_{1}(\xi)\left(M_{T 1}^{2}-2\right) \xi \cosh \left(\alpha_{1} \xi y\right) \\
& -m_{3}(\xi) g_{2}(\xi)\left(M_{T 1}^{2}-2\right) \xi \sinh \left(\alpha_{1} \xi y\right) \\
& \left.-m_{3}(\xi) \times 2 \beta_{1} \xi \cosh \left(\beta_{1} \xi y\right)\right\} \\
& \times J_{2 n-1}(\xi a) \cos (\xi x) d \xi \\
+\sum_{n=1}^{\infty} d_{n}(2 n) / \pi \times \int_{0}^{\infty}\left\{m_{2}(\xi)\right. & f_{1}(\xi)\left(M_{T 1}^{2}-2\right) \xi \cosh \left(\alpha_{1} \xi y\right) \\
& +m_{2}(\xi) f_{2}(\xi)\left(M_{T 1}^{2}-2\right) \xi \sinh \left(\alpha_{1} \xi y\right) \\
& +m_{2}(\xi) \times 2 \beta_{1} \xi \sinh \left(\beta_{1} \xi y\right) \\
& -m_{4}(\xi) g_{1}(\xi)\left(M_{T 1}^{2}-2\right) \xi \cosh \left(\alpha_{1} \xi y\right) \\
& -m_{4}(\xi) g_{2}(\xi)\left(M_{T 1}^{2}-2\right) \xi \sinh \left(\alpha_{1} \xi y\right) \\
& \left.-m_{4}(\xi) \times 2 \beta_{1} \xi \cosh \left(\beta_{1} \xi y\right)\right\} \\
& \times J_{2 n}(\xi a) \cos (\xi x) d \xi
\end{aligned}
$$

where the expressions of known functions $m_{1}(\xi) m_{2}(\xi), m_{3}(\xi)$ and $m_{4}(\xi)$ are shown in Appendix E.

As long as $\cosh \left(\alpha_{1} \xi y\right), \sinh \left(\alpha_{1} \xi y\right), \cosh \left(\beta_{1} \xi y\right)$ and $\sinh \left(\beta_{1} \xi y\right)$ are contained in the integrands in Eq. (36), Eq. (34) can not be applied, and thus the circumferential stress intensity factor $K_{\theta \theta}$ can not be determined. This led to the initially difficulty in solving the present problem. However, if the stress singularity is evaluated using Eq. (34), the integrands in Eq. (36) must be expressed in the same form as that in Eq. (34) for a large $\xi$. Therefore, the author attempted to calculate the functions $f_{1}(\xi), f_{2}(\xi), g_{1}(\xi), g_{2}(\xi), m_{1}$ $(\xi), m_{2}(\xi), m_{3}(\xi)$ and $m_{4}(\xi)$. As a result, the following relations were found to be valid for a large $\xi$,

$$
\begin{aligned}
& m_{1}(\xi)=m_{3}(\xi), \quad m_{2}(\xi)=m_{4}(\xi) \\
& f_{1}(\xi)-g_{1}(\xi)=-\left[f_{2}(\xi)-g_{2}(\xi)\right]
\end{aligned}
$$

Therefore, Eq. (36) can be rewritten as follow

$$
\begin{aligned}
\tau_{y y 1}^{0}=\sum_{n=1}^{\infty} c_{n}(2 n-1) / \pi \times \int_{0}^{\infty}\{ & p_{1}(\xi)\left[\cosh \left(\alpha_{1} \xi y\right)-\sinh \left(\alpha_{1} \xi y\right)\right] \\
+ & \left.p_{2}(\xi)\left[\cosh \left(\beta_{1} \xi y\right)-\sinh \left(\beta_{1} \xi y\right)\right]\right\} \\
& \times J_{2 n-1}(\xi a) \cos (\xi x) d \xi \\
+\sum_{n=1}^{\infty} d_{n}(2 n) / \pi \times \int_{0}^{\infty}\left\{p_{3}(\xi)[\right. & \left.\left.\cosh \left(\alpha_{1} \xi y\right)-\sinh \left(\alpha_{1} \xi y\right)\right]\right\} \\
+ & \left.p_{4}(\xi)\left[\cosh \left(\beta_{1} \xi y\right)-\sinh \left(\beta_{1} \xi y\right)\right]\right\} \\
& \times J_{2 n}(\xi a) \cos (\xi x) d \xi
\end{aligned}
$$

with

$$
\begin{array}{ll}
p_{1}(\xi)=m_{1}(\xi)\left[f_{1}(\xi)-g_{1}(\xi)\right]\left(M_{T 1}^{2}-2\right) \xi, & p_{2}(\xi)=-m_{1}(\xi) \times 2 \beta_{1} \xi \\
p_{3}(\xi)=m_{2}(\xi)\left[f_{1}(\xi)-g_{1}(\xi)\right]\left(M_{T 1}^{2}-2\right) \xi, & p_{4}(\xi)=-m_{2}(\xi) \times 2 \beta_{1} \xi
\end{array}
$$

In order to extract the stress singularity, Eq. (38) is once more changed into the form

$$
\begin{aligned}
& \tau_{y y 1}^{0}=\sum_{n=1}^{\infty} c_{n}(2 n-1) / \pi \times \int_{0}^{\infty}\left\{p_{1}(\xi)\left[\cosh \left(\alpha_{1} \xi y\right)-\sinh \left(\alpha_{1} \xi y\right)\right]\right. \\
&- p_{1}^{L} \exp \left(-\alpha_{1} \xi y\right)+p_{2}(\xi)\left[\cosh \left(\beta_{1} \xi y\right)\right. \\
&-\left.\left.\sinh \left(\beta_{1} \xi y\right)\right]-p_{2}^{L} \exp \left(-\beta_{1} \xi y\right)\right\} \\
& \times J_{2 n-1}(\xi a) \cos (\xi x) d \xi
\end{aligned}
$$

$$
\begin{aligned}
& +\sum_{n=1}^{\infty} d_{n}(2 n) / \pi \times \int_{0}^{\infty}\left\{p_{3}(\xi)\left[\cosh \left(\alpha_{1} \xi y\right)-\sinh \left(\alpha_{1} \xi y\right)\right]\right. \\
& -p_{3}^{L} \exp \left(-\alpha_{1} \xi y\right)+p_{4}(\xi)\left[\cosh \left(\beta_{1} \xi y\right)\right. \\
& \left.\left.-\sinh \left(\beta_{1} \xi y\right)\right]-p_{4}^{L} \exp \left(-\beta_{1} \xi y\right)\right\} \\
& \times J_{2 n}(\xi a) \cos (\xi x) d \xi \\
& +\sum_{n=1}^{\infty} c_{n}(2 n-1) / \pi \times\left\{(-1)^{n} p_{1}^{L}\left[\cos \theta+\left(\cos ^{2} \theta+\alpha_{1}^{2} \sin ^{2} \theta\right)^{1 / 2}\right]^{1 / 2}\right. \\
& /\left[4 \operatorname{ar}\left(\cos ^{2} \theta+\alpha_{1}^{2} \sin ^{2} \theta\right)^{1 / 2}\right]^{1 / 2} \\
& +(-1)^{n} p_{2}^{L}\left[\cos \theta+\left(\cos ^{2} \theta+\alpha_{1}^{2} \sin ^{2} \theta\right)^{1 / 2}\right]^{1 / 2} \\
& \left./\left[4 \operatorname{ar}\left(\cos ^{2} \theta+\alpha_{1}^{2} \sin ^{2} \theta\right)^{1 / 2}\right]^{1 / 2}\right\} \\
& \begin{array}{r}
+\sum_{n=1}^{\infty} d_{n}(2 n) / \pi \times\left\{(-1)^{n} p_{3}^{L}\left[-\cos \theta+\left(\cos ^{2} \theta+\alpha_{1}^{2} \sin ^{2} \theta\right)^{1 / 2}\right]^{1 / 2}\right. \\
\quad /\left[4 \operatorname{ar}\left(\cos ^{2} \theta+\alpha_{1}^{2} \sin ^{2} \theta\right)^{1 / 2}\right]^{1 / 2} \\
+(-1)^{n} p_{4}^{L}\left[-\cos \theta+\left(\cos ^{2} \theta+\alpha_{1}^{2} \sin ^{2} \theta\right)^{1 / 2}\right]^{1 / 2} \\
\left./\left[4 \operatorname{ar}\left(\cos ^{2} \theta+\alpha_{1}^{2} \sin ^{2} \theta\right)^{1 / 2}\right]^{1 / 2}\right\}
\end{array}
\end{aligned}
$$

where constants $p_{i}^{L}(i=1,2,3,4)$ can be calculated by

$$
p_{i}^{L}=p_{i}\left(\xi_{L}\right)
$$

In the same manner, the singularities are extracted from the stress expressions of $\tau_{x x 1}, \tau_{x y 1}, \tau_{y y 2}, \tau_{x x 2}, \tau_{x y 2}$. Finally, the circumferential stress intensity factor can be determined by

$$
\begin{aligned}
& K_{\theta \theta}=\left.\sqrt{2 \pi r} \tau_{\theta \theta 1}\right|_{r \rightarrow 0} \\
& =\sum_{n=1}^{\infty} c_{n}(2 n-1) \mu_{1} \times(-1)^{n} /(2 \pi a)^{1 / 2} \\
& \times\left\{\left(p_{1}^{L} \cos ^{2} \theta+p_{5}^{L} \sin ^{2} \theta\right)\left[\cos \theta+\left(\cos ^{2} \theta+\alpha_{1}^{2} \sin ^{2} \theta\right)^{1 / 2}\right]^{1 / 2}\right. \\
& /\left(\cos ^{2} \theta+\alpha_{1}^{2} \sin ^{2} \theta\right)^{1 / 2} \\
& +\left(p_{2}^{L} \cos ^{2} \theta+p_{6}^{L} \sin ^{2} \theta\right)\left[\cos \theta+\left(\cos ^{2} \theta+\beta_{1}^{2} \sin ^{2} \theta\right)^{1 / 2}\right]^{1 / 2} \\
& /\left(\cos ^{2} \theta+\beta_{1}^{2} \sin ^{2} \theta\right)^{1 / 2} \\
& +2 p_{9}^{L} \sin \theta \cos \theta\left[-\cos \theta+\left(\cos ^{2} \theta+\alpha_{1}^{2} \sin ^{2} \theta\right)^{1 / 2}\right]^{1 / 2} \\
& /\left(\cos ^{2} \theta+\alpha_{1}^{2} \sin ^{2} \theta\right)^{1 / 2} \\
& +2 p_{10}^{L} \operatorname{con} i n \theta \cos \theta\left[-\cos \theta+\left(\cos ^{2} \theta+\beta_{1}^{2} \sin ^{2} \theta\right)^{1 / 2}\right]^{1 / 2} \\
& \left./\left(\cos ^{2} \theta+\beta_{1}^{2} \sin ^{2} \theta\right)^{1 / 2}\right\} \\
& \begin{array}{l}
+\sum_{n=1}^{\infty} d_{n}(2 n) \mu_{1} \times(-1)^{n} /(2 \pi a)^{1 / 2} \\
\quad \times\left\{\left(p_{3}^{L} \cos ^{2} \theta+p_{7}^{L} \sin ^{2} \theta\right)-\cos \theta+\left(\cos ^{2} \theta+\alpha_{1}^{2} \sin ^{2} \theta\right)^{1 / 2}\right]^{1 / 2} \\
\quad /\left(\cos ^{2} \theta+\alpha_{1}^{2} \sin ^{2} \theta\right)^{1 / 2}
\end{array} \\
& +\left(p_{4}^{L} \cos ^{2} \theta+p_{8}^{L} \sin ^{2} \theta\right)-\left[\cos \theta+\left(\cos ^{2} \theta+\beta_{1}^{2} \sin ^{2} \theta\right)^{1 / 2}\right]^{1 / 2} \\
& /\left(\cos ^{2} \theta+\beta_{1}^{2} \sin ^{2} \theta\right)^{1 / 2} \\
& \left.\left.-2 p_{11}^{L} \sin \theta \cos \theta\right)\left[\cos \theta+\alpha_{1}^{2} \sin ^{2} \theta\right)^{1 / 2}\right]^{1 / 2} \\
& /\left(\cos ^{2} \theta+\alpha_{1}^{2} \sin ^{2} \theta\right)^{1 / 2} \\
& -2 p_{12}^{L} \operatorname{con} i n \theta \cos \theta\left[\cos \theta+\left(\cos ^{2} \theta+\beta_{1}^{2} \sin ^{2} \theta\right)^{1 / 2}\right]^{1 / 2} \\
& \left./\left(\cos ^{2} \theta+\beta_{1}^{2} \sin ^{2} \theta\right)^{1 / 2}\right\}
\end{aligned}
$$$$
\text { for } 0 \leq \theta \leq \pi
$$

$$
\begin{aligned}
& K_{\theta \theta}=\left.\sqrt{2 \pi r} \tau_{\theta \theta 2}\right|_{r \rightarrow 0} \\
& +\sum_{n=1}^{\infty} c_{n}(2 n-1) \mu_{2} \times(-1)^{n} /(2 \pi a)^{1 / 2} \\
& \times\left\{\left(p_{13}^{L} \cos ^{2} \theta+p_{14}^{L} \sin ^{2} \theta\right)\left[\cos \theta+\left(\cos ^{2} \theta+\alpha_{2}^{2} \sin ^{2} \theta\right)^{1 / 2}\right]^{1 / 2}\right. \\
& \quad /\left(\cos ^{2} \theta+\alpha_{2}^{2} \sin ^{2} \theta\right)^{1 / 2} \\
& +\left(p_{15}^{L} \cos ^{2} \theta+p_{16}^{L} \sin ^{2} \theta\right)\left[\cos \theta+\left(\cos ^{2} \theta+\beta_{2}^{2} \sin ^{2} \theta\right)^{1 / 2}\right]^{1 / 2} \\
& \quad /\left(\cos ^{2} \theta+\beta_{2}^{2} \sin ^{2} \theta\right)^{1 / 2} \\
& \left.\left.+2 p_{17}^{L} \sin \theta \cos \theta\right)\left[-\cos \theta+\alpha_{2}^{2} \sin ^{2} \theta\right)^{1 / 2}\right]^{1 / 2} \\
& \quad /\left(\cos ^{2} \theta+\alpha_{2}^{2} \sin ^{2} \theta\right)^{1 / 2} \\
& \left.+2 p_{18 . \operatorname{con} i n \theta \cos \theta}^{L}-\cos \theta+\left(\cos ^{2} \theta+\beta_{2}^{2} \sin ^{2} \theta\right)^{1 / 2}\right]^{1 / 2} \\
& \left.\quad /\left(\cos \theta+\beta_{2}^{2} \sin ^{2} \theta\right)^{1 / 2}\right\}
\end{aligned}
$$




$$
\begin{gathered}
+\sum_{n=1}^{\infty} d_{n}(2 n) \mu_{2} \times(-1)^{n} /(2 \pi a)^{1 / 2} \\
\times\left\{\left(p_{19}^{L} \cos ^{2} \theta+p_{20}^{L} \sin ^{2} \theta\right)\left[-\cos \theta+\left(\cos ^{2} \theta+\alpha_{2}^{2} \sin ^{2} \theta\right)^{1 / 2}\right]^{1 / 2}\right. \\
/\left(\cos ^{2} \theta+\alpha_{2}^{2} \sin ^{2} \theta\right)^{1 / 2} \\
+\left(p_{21}^{L} \cos ^{2} \theta+p_{22}^{L} \sin ^{2} \theta\right)\left[-\cos \theta+\left(\cos ^{2} \theta+\beta_{2}^{2} \sin ^{2} \theta\right)^{1 / 2}\right]^{1 / 2} \\
/\left(\cos ^{2} \theta+\beta_{2}^{2} \sin ^{2} \theta\right)^{1 / 2} \\
-2 p_{23}^{L} \sin \theta \cos \theta\left[\cos \theta+\left(\cos ^{2} \theta+\alpha_{2}^{2} \sin ^{2} \theta\right)^{1 / 2}\right]^{1 / 2} \\
/\left(\cos ^{2} \theta+\alpha_{2}^{2} \sin ^{2} \theta\right)^{1 / 2} \\
-2 p_{24}^{L} \operatorname{con} i n \theta \cos \theta\left[\cos \theta+\left(\cos ^{2} \theta+\beta_{2}^{2} \sin ^{2} \theta\right)^{1 / 2}\right]^{1 / 2} \\
\left./\left(\cos ^{2} \theta+\beta_{2}^{2} \sin ^{2} \theta\right)^{1 / 2}\right\} \\
\text { for }-\pi \leq \theta \leq 0
\end{gathered}
$$

where $p_{i}^{L}(i=5,6, \ldots, 24)$ can be calculated by Eq. (41). Here, the expressions of known functions $p_{i}(i=5,6, \ldots, 24)$ are shown in Appendix F.

\section{Numerical Examples and Results}

We consider the case in which an infinite layer made of epoxy resin is sandwiched between two elastic half-planes made of aluminum. The material constants of these materials are listed in Table 1. Through numerical calculations, it was verified that the convergence of the integrands in Eq. (31) is superior and that the accuracy of the Schmidt method in solving Eq. (30) is satisfactory.

First, the present numerical results are compared with those given by Chen, Keer and Achenbach [5]. For this purpose, $K_{\theta \theta} /(p$ $\sqrt{\pi a}$ ) is calculated for $\rho_{1}=\rho_{2}=\rho_{3}=\rho_{4}, \nu_{1}=\nu_{2}=\nu_{3}=\nu_{4}=0.3, \mu_{1}=$ $\mu_{2}=\mu_{4}, \mu_{3} / \mu_{1}=0.8, h_{1} / a=0.4, h_{2} / a=0.4$ and $M_{T 1}=0.8$. The results of this calculation are shown in Table 2 . The values in parentheses are those picked from a curve provided in Ref. [5]. The table reveals that the present analysis is appropriate.

In Figs. 3, 4 and 5, the values of the circumferential stress intensity factor $K_{\theta \theta} /(p \sqrt{\pi a})$ are plotted for $\left(h_{1}+h_{2}\right) / a=2.0$. Figures 6 , 7 and 8 show the curves for $\left(h_{1}+h_{2}\right) / a=1.0$. The figures show that the stress intensity factor $K_{\theta \theta}$ declines as the $\left(h_{1}+h_{2}\right) / a$ ratio decreases. When the propagation velocity of the moving crack increases, the crack tends to become curved, as pointed out by Yoffe [3]. If the crack approaches the interface $y=h_{1}$, the peak value of $K_{\theta \theta}$ appears at $\theta \leq 0$. This means that a moving crack with a high speed may propagate toward the opposite interface $y=$ $-h_{2}$. Namely, the crack may have a tendency to propagate in a

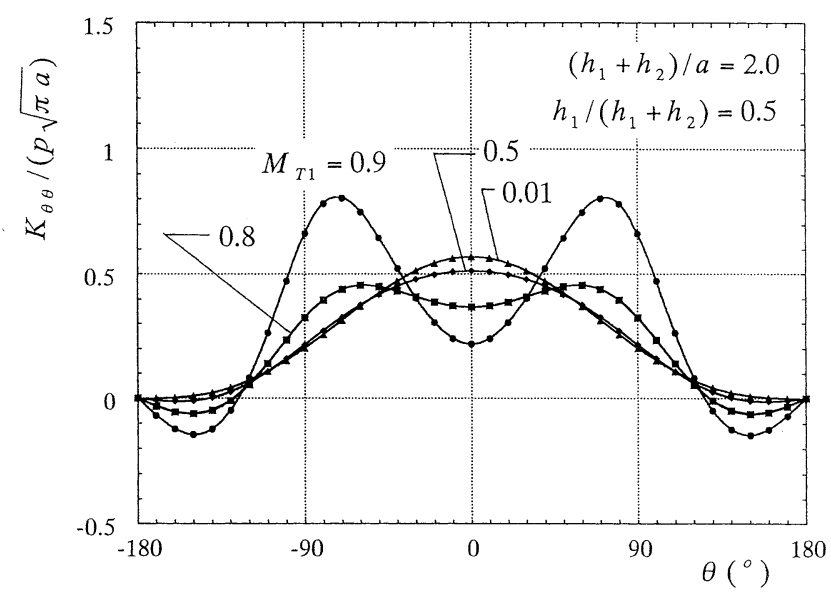

Figure 3 Stress intensity factor $K_{\theta \theta} /(p \sqrt{\pi a})$ for $\left(h_{1}+h_{2}\right) / a=2.0$ and $h_{1} /$ $\left(h_{1}+h_{2}\right)=0.5$.
Table 1 Material constants.

\begin{tabular}{lcc}
\hline \hline Constants & Aluminum & Epoxy Resin \\
\hline$\mu(G P a)$ & 26.42 & 1.144 \\
$v$ & 0.3 & 0.35 \\
$\rho\left(10^{3} \mathrm{~kg} / \mathrm{m}^{3}\right)$ & 2.69 & 1.40 \\
\hline
\end{tabular}

Table 2 Stress intensity factor $K_{\theta \theta} /(p \sqrt{\pi a})$ for two dissimilar bonded half-planes weakened by a crack $\left(\rho_{1}=\rho_{2}=\rho_{3}=\rho_{4}, \nu_{1}=\nu_{2}=\nu_{3}=\right.$ $\nu_{4}=0.3, \mu_{1}=\mu_{2}=\mu_{4}, \mu_{3} / \mu_{1}=0.8, h_{1} / a=0.4, h_{2} / a=0.4$ and $M_{T 1}=$ $0.8)$.

\begin{tabular}{cccccccc}
\hline$\theta\left({ }^{\circ}\right)$ & -180 & -120 & -60 & 0 & 60 & 120 & 180 \\
\hline$K_{\theta \theta}$ & -0.000 & -0.297 & 1.181 & 1.254 & 2.229 & 0.736 & 0.000 \\
$/(p \sqrt{\pi a})$ & $(-0.005)$ & $(-0.292)$ & $(1.184)$ & $(1.251)$ & $(2.223)$ & $(0.703)$ & $(-0.000)$ \\
\hline
\end{tabular}

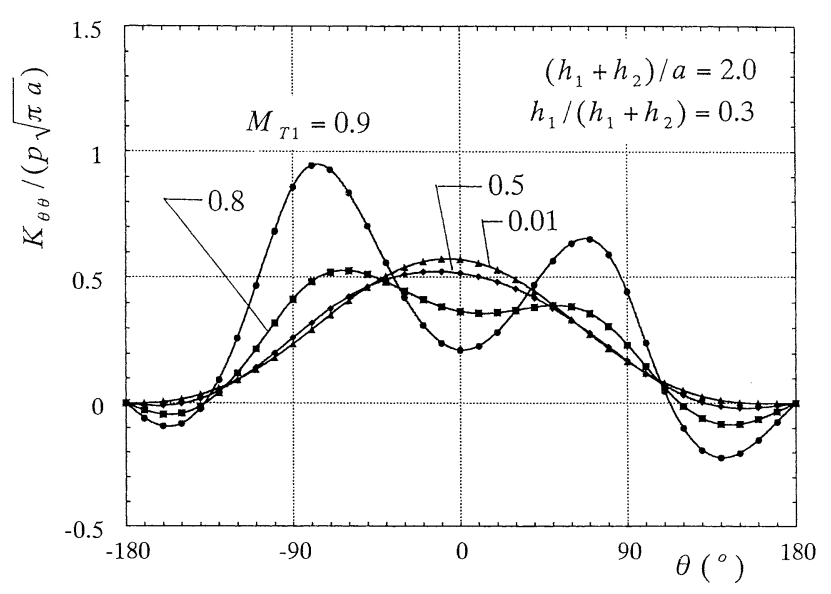

Figure 4 Stress intensity factor $K_{\theta \theta} /(p \sqrt{\pi a})$ for $\left(h_{1}+h_{2}\right) / a=2.0$ and $h_{1} /$ $\left(h_{1}+h_{2}\right)=0.3$.

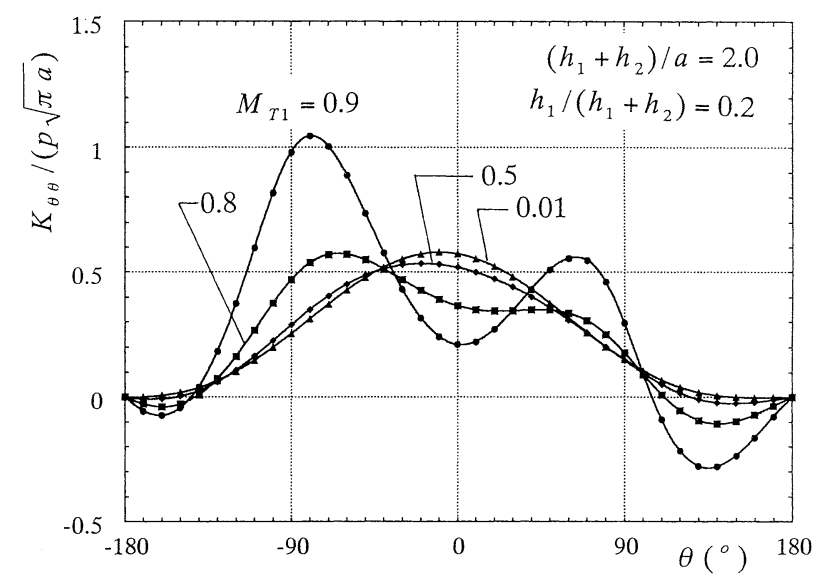

Figure 5 Stress intensity factor $K_{\theta \theta} /(p \sqrt{\pi a})$ for $\left(h_{1}+h_{2}\right) / a=2.0$ and $h_{1} /$ $\left(h_{1}+h_{2}\right)=0.2$. 


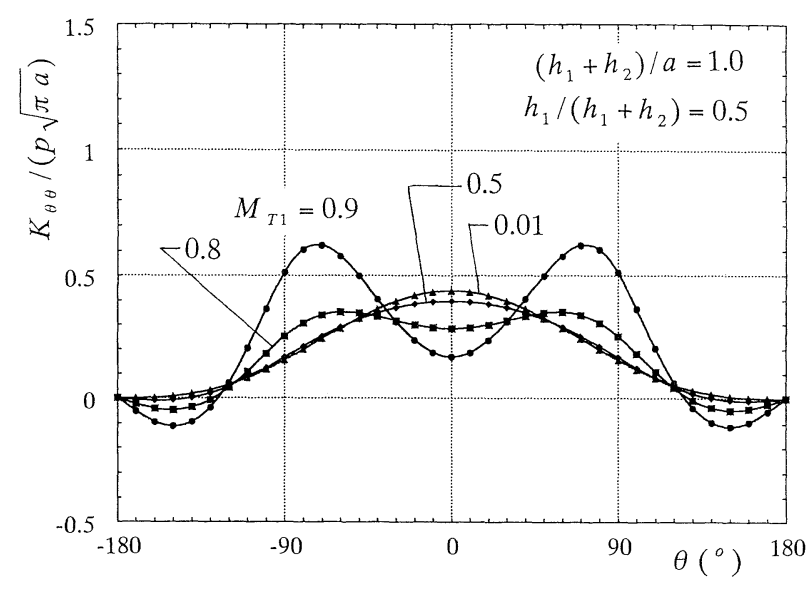

Figure 6 Stress intensity factor $K_{\theta \theta} /(p \sqrt{\pi a})$ for $\left(h_{1}+h_{2}\right) / a=1.0$ and $h_{1} /$ $\left(h_{1}+h_{2}\right)=0.5$.

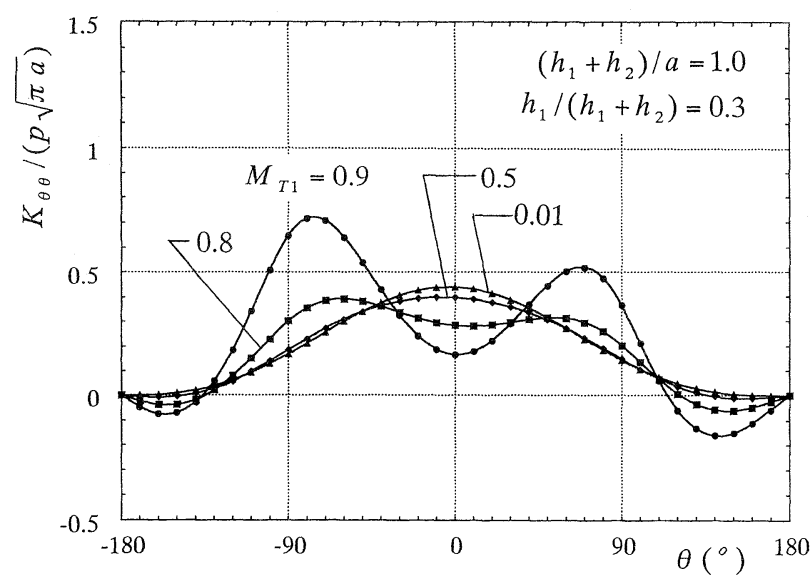

Figure 7 Stress intensity factor $K_{\theta \theta} /(p \sqrt{\pi a})$ for $\left(h_{1}+h_{2}\right) / a=1.0$ and $h_{1} /$ $\left(h_{1}+h_{2}\right)=0.3$.

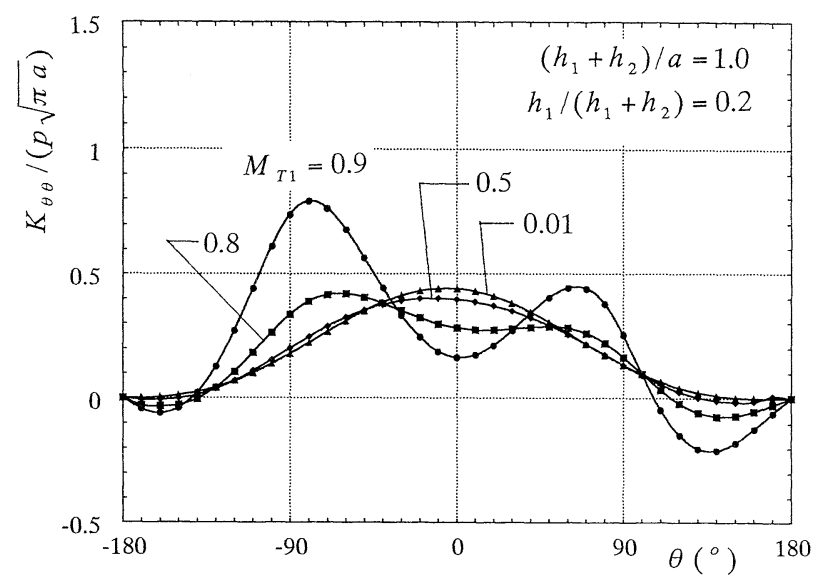

Figure 8 Stress intensity factor $K_{\theta \theta} /(p \sqrt{\pi a})$ for $\left(h_{1}+h_{2}\right) / a=1.0$ and $h_{1} /$ $\left(h_{1}+h_{2}\right)=0.2$. wavelike manner near the mid-plane $y=\left(h_{1}-h_{2}\right) / 2$, as shown in Fig. 9.

Appendix A

$a_{11}^{(1)}=f_{1}(\xi) \xi, \quad a_{12}^{(1)}=g_{1}(\xi) \xi-\beta_{1} \xi, \quad a_{21}^{(1)}=f_{2}(\xi) a_{1} \xi-\xi$, $a_{22}^{(1)}=g_{2}(\xi) a_{1} \xi$

$a_{11}^{(2)}=f_{1}^{\prime}(\xi) \xi, \quad a_{12}^{(2)}=g_{1}^{\prime}(\xi) \xi-\beta_{2} \xi, \quad a_{21}^{(2)}=f_{2}^{\prime}(\xi) a_{2} \xi-\xi$, $a_{22}^{(2)}=g_{2}^{\prime}(\xi) a_{2} \xi$

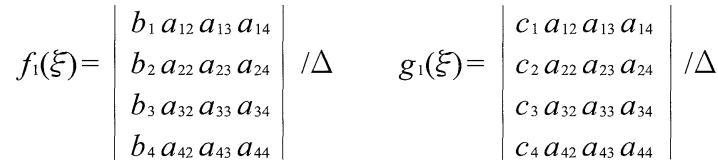

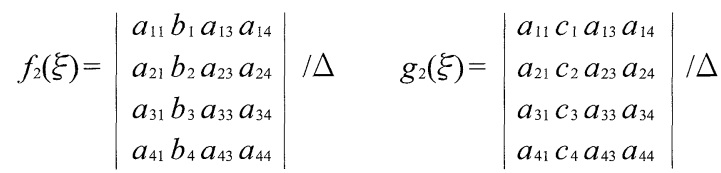

$$
\begin{aligned}
& \Delta=\left|a_{i j}\right|, \quad(i=1, \ldots, 4), \quad(j=1, \ldots, 4)
\end{aligned}
$$

$a_{11}=-\xi^{2}\left(M_{T 1}^{2}-2\right) \cosh \left(\alpha_{1} \xi h_{1}\right) \mu_{1}, a_{12}=-\xi^{2}\left(M_{T 1}^{2}-2\right) \sinh \left(\alpha_{1} \xi h_{1}\right) \mu_{1}$, $a_{13}=\xi^{2}\left(M_{T 3}^{2}-2\right) \exp \left(-\alpha_{3}|\xi| h_{1}\right) \mu_{3}, a_{14}=-2 \xi|\xi| \beta_{3} \exp \left(-\beta_{3}|\xi| h_{1}\right) \mu_{3}$, $b_{1}=2 \beta_{1} \xi^{2} \sinh \left(\beta_{1} \xi h_{1}\right) \mu_{1}, c_{1}=2 \beta_{1} \xi^{2} \cosh \left(\beta_{1} \xi h_{1}\right) \mu_{1}$,

$a_{21}=-2 \alpha_{1} \xi^{2} \sinh \left(\alpha_{1} \xi h_{1}\right) \mu_{1}, a_{22}=-2 \alpha_{1} \xi^{2} \cosh \left(\alpha_{1} \xi h_{1}\right) \mu_{1}$, $a_{23}=-2 \xi|\xi| \beta_{3} \exp \left(-\alpha_{3}|\xi| h_{1}\right) \mu_{3}, a_{24}=\left(M_{T_{3}}^{2}-2\right) \xi^{2} \exp \left(-\beta_{3}|\xi| h_{1}\right) \mu_{3}$, $b_{2}=\left(M_{T 1}^{2}-2\right) \xi^{2} \cosh \left(\beta_{1} \xi h_{1}\right) \mu_{1}, c_{2}=\left(M_{T 1}^{2}-2\right) \xi^{2} \sinh \left(\beta_{1} \xi h_{1}\right) \mu_{1}$,

$a_{31}=-\xi \cosh \left(\alpha_{1} \xi h_{1}\right), a_{32}=-\xi \sinh \left(\alpha_{1} \xi h_{1}\right), a_{33}=\xi \exp \left(-\alpha_{3}|\xi| h_{1}\right)$, $a_{34}=|\xi| \beta_{3} \exp \left(-\beta_{3}|\xi| h_{1}\right), b_{3}=-\beta_{1} \xi \sinh \left(\beta_{1} \xi h_{1}\right), c_{3}=-\beta_{1} \xi \cosh$ $\left(\beta_{1} \xi h_{1}\right)$

$a_{41}=\alpha_{1} \xi \sinh \left(\alpha_{1} \xi h_{1}\right), a_{42}=\alpha_{1} \xi \cosh \left(\alpha_{1} \xi h_{1}\right), a_{34}=|\xi| \beta_{3} \exp \left(-\alpha_{3}|\xi| h_{1}\right)$, $a_{44}=\xi \exp \left(-\beta_{3}|\xi| h_{1}\right), b_{4}=\xi \cosh \left(\beta_{1} \xi h_{1}\right), c_{4}=\xi \sinh \left(\beta_{1} \xi h_{1}\right)$

$$
\begin{aligned}
& f_{1}^{\prime}(\xi)=\left|\begin{array}{llll}
b_{1}^{\prime} & a_{12}^{\prime} & a_{13}^{\prime} & a_{14}^{\prime} \\
b_{2}^{\prime} & a_{22}^{\prime} & a_{23}^{\prime} & a_{24}^{\prime} \\
b_{3}^{\prime} & a_{32}^{\prime} & a_{33}^{\prime} & a_{34}^{\prime} \\
b_{4}^{\prime} & a_{42}^{\prime} & a_{43}^{\prime} & a_{44}^{\prime}
\end{array}\right| / \Delta^{\prime}, \quad g_{1}^{\prime}(\xi)=\left|\begin{array}{cccc}
c_{1}^{\prime} & a_{12}^{\prime} & a_{13}^{\prime} & a_{14}^{\prime} \\
c_{2}^{\prime} & a_{22}^{\prime} & a_{23}^{\prime} & a_{24}^{\prime} \\
c_{3}^{\prime} & a_{32}^{\prime} & a_{33}^{\prime} & a_{34}^{\prime} \\
c_{4}^{\prime} & a_{42}^{\prime} & a_{43}^{\prime} & a_{44}^{\prime}
\end{array}\right| / \Delta^{\prime} \\
& f_{2}^{\prime}(\xi)=\left|\begin{array}{llll}
a_{11}^{\prime} & b_{1}^{\prime} & a_{13}^{\prime} & a_{14}^{\prime} \\
b_{21}^{\prime} & b_{2}^{\prime} & a_{23}^{\prime} & a_{24}^{\prime} \\
a_{31}^{\prime} & b_{3}^{\prime} & a_{33}^{\prime} & a_{34}^{\prime} \\
a_{41}^{\prime} & b_{4}^{\prime} & a_{43}^{\prime} & a_{44}^{\prime}
\end{array}\right| / \Delta^{\prime}, \quad g_{2}^{\prime}(\xi)=\left|\begin{array}{llll}
a_{11}^{\prime} & c_{1}^{\prime} & a_{13}^{\prime} & a_{14}^{\prime} \\
a_{21}^{\prime} & c_{2}^{\prime} & a_{23}^{\prime} & a_{24}^{\prime} \\
a_{31}^{\prime} & c_{3}^{\prime} & a_{33}^{\prime} & a_{34}^{\prime} \\
a_{41}^{\prime} & c_{4}^{\prime} & a_{43}^{\prime} & a_{44}^{\prime}
\end{array}\right| / \Delta
\end{aligned}
$$

Figure 9 Possible shape of a moving crack. 


$$
\begin{aligned}
& \Delta^{\prime}=\left|a_{i j}\right|, \quad(i=1, \ldots, 4), \quad(j=1, \ldots, 4) \\
& a_{11}^{\prime}=-\xi^{2}\left(M_{T 2}^{2}-2\right) \cosh \left(-\alpha_{2} \xi h_{2}\right) \mu_{2}, a_{12}^{\prime}=-\xi^{2}\left(M_{T 2}^{2}-2\right) \sinh \left(-\alpha_{2} \xi h_{2}\right) \\
& \mu_{2}, \\
& a_{13}^{\prime}=\xi^{2}\left(M_{T 4}^{2}-2\right) \exp \left(-\alpha_{4}|\xi| h_{2}\right) \mu_{4}, a_{14}^{\prime}=2 \xi|\xi| \beta_{4} \exp \left(-\beta_{43}|\xi| h_{2}\right) \mu_{4}, \\
& b_{1}^{\prime}=2 \beta_{2} \xi^{2} \sinh \left(-\beta_{2} \xi h_{2}\right) \mu_{2}, c_{1}^{\prime}=2 \beta_{2} \xi^{2} \cosh \left(-\beta_{2} \xi h_{2}\right) \mu_{2},
\end{aligned}
$$

Appendix B

$k_{1}^{(1)}=-p_{1} a_{22}^{(1)} / \Delta_{(1)}+p_{2} a_{21}^{(1)} / \Delta_{(1)}, k_{2}^{(1)}=-p_{1} a_{12}^{(1)} / \Delta_{(1)}+p_{2} a_{11}^{(1)} / \Delta_{(1)}$, $k_{3}^{(1)}=p_{3} a_{22}^{(1)} / \Delta_{(1)}-p_{4} a_{21}^{(1)} / \Delta_{(1)}, \quad k_{4}^{(1)}=p_{3} a_{12}^{(1)} / \Delta_{(1)}-p_{4} a_{11}^{(1)} / \Delta_{(1)}$,

$p_{1}=-\mu_{1} f_{1}(\xi)\left(M_{T 1}^{2}-2\right), \quad p_{2}=-\mu_{1} \xi^{2}\left\{g_{1}(\xi)\left(M_{T 1}^{2}-2\right)+2 \beta_{1}\right\}$, $p_{3}=\mu_{1} \xi^{2}\left\{2 \alpha_{1} f_{2}(\xi)+\left(M_{T 1}^{2}-2\right)\right\}, \quad p_{4}=\mu_{1} \xi^{2} \times 2 \alpha_{1} \xi^{2} g_{2}(\xi)$

$$
\Delta_{(1)}=a_{11}^{(1)} a_{22}^{(1)}-a_{12}^{(1)} a_{12}^{(1)}
$$

$k_{1}^{(2)}=-p_{1}^{\prime} a_{22}^{(2)} / \Delta_{(2)}+p_{2}^{\prime} a_{21}^{(2)} / \Delta_{(2)}, k_{2}^{(2)}=-p_{1}^{\prime} a_{12}^{(2)} / \Delta_{(2)}+p_{2}^{\prime} a_{11}^{(2)} / \Delta_{(2)}$, $k_{3}^{(2)}=p_{3}^{\prime} a_{22}^{(2)} / \Delta_{(2)}-p_{4}^{\prime} a_{21}^{(2)} / \Delta_{(2)}, k_{4}^{(2)}=p_{3}^{\prime} a_{12}^{(2)} / \Delta_{(2)}-p_{4}^{\prime} a_{11}^{(2)} / \Delta_{(2)}$,

$p_{1}^{\prime}=-\mu_{2} f_{1}^{\prime}(\xi)\left(M_{T 2}^{2}-2\right), \quad p_{2}^{\prime}=-\mu_{2} \xi^{2}\left\{g_{1}^{\prime}(\xi)\left(M_{T 2}^{2}-2\right)+2 \beta_{21}\right\}$, $p_{3}^{\prime}=\mu_{2} \xi^{2}\left\{2 \alpha_{21} f_{2}^{\prime}(\xi)+\left(M_{T 2}^{2}-2\right)\right\}, \quad p_{4}^{\prime}=\mu_{1} \times 2 \alpha_{21} \xi^{2} g_{2}^{\prime}(\xi)$

$$
\Delta_{(2)}=a_{11}^{(2)} a_{22}^{(2)}-a_{12}^{(2)} a_{12}^{(2)}
$$

\section{Appendix C}

$l_{1}(\xi)=\left\{k_{1}^{(2)} k_{4}^{(1)}-k_{3}^{(2)} k_{2}^{(1)}\right\} / \Delta^{\prime}, \quad l_{2}(\xi)=\left\{k_{2}^{(2)} k_{4}^{(1)}-k_{4}^{(2)} k_{2}^{(1)}\right\} / \Delta^{\prime}$, $l_{3}(\xi)=\left\{k_{1}^{(1)} k_{3}^{(2)}-k_{3}^{(1)} k_{1}^{(2)}\right\} / \Delta^{\prime}, \quad l_{4}(\xi)=\left\{k_{1}^{(1)} k_{4}^{(2)}-k_{3}^{(1)} k_{2}^{(2)}\right\} / \Delta^{\prime}$, $\Delta^{\prime}=k_{1}^{(1)} k_{4}^{(1)}-k_{3}^{(1)} k_{2}^{(1)}$

Appendix D $Q_{1}(\xi)=\left\{l_{2}(\xi) k_{1}^{(2)}-\left[l_{1}(\xi)-1\right] k_{2}^{(2)}\right\} / \Delta^{\prime \prime}$,

$$
\begin{aligned}
& Q_{2}(\xi)=\left\{l_{3}(\xi) k_{2}^{(2)}-\left[l_{4}(\xi)-1\right] k_{1}^{(2)}\right\} / \Delta^{\prime \prime}, \\
& Q_{3}(\xi)=\left\{l_{2}(\xi) k_{3}^{(2)}-\left[l_{1}(\xi)-1\right] k_{4}^{(2)}\right\} / \Delta^{\prime \prime}, \\
& Q_{4}(\xi)=\left\{l_{3}(\xi) k_{2}^{(2)}-\left[l_{4}(\xi)-1\right] k_{3}^{(2)}\right\} / \Delta^{\prime \prime}, \\
& \Delta^{\prime \prime}=l_{3}(\xi) l_{2}(\xi)-\left[l_{1}(\xi)-1\right]\left[l_{4}(\xi)-1\right]
\end{aligned}
$$

$$
\begin{aligned}
& \text { Appendix E } \\
& \begin{aligned}
m_{1}(\xi) & =\left\{l_{2}(\xi) a_{22}^{(1)}+\left[l_{2}(\xi) l_{3}(\xi)-l_{1}(\xi) l_{4}(\xi)+l_{4}(\xi)\right] a_{12}^{(1)}\right\} /\left(\Delta^{\prime \prime} \Delta_{(1)}\right) \\
m_{2}(\xi) & =\left\{l_{3}(\xi) a_{12}^{(1)}+\left[l_{2}(\xi) l_{3}(\xi)-l_{1}(\xi) l_{4}(\xi)+l_{1}(\xi)\right] a_{22}^{(1)}\right\} /\left(\Delta^{\prime \prime} \Delta_{(1)}\right) \\
m_{3}(\xi) & =\left\{l_{2}(\xi) a_{21}^{(1)}+\left[l_{2}(\xi) l_{3}(\xi)-l_{1}(\xi) l_{4}(\xi)+l_{4}(\xi)\right] a_{11}^{(1)}\right\} /\left(\Delta^{\prime \prime} \Delta_{(1)}\right) \\
m_{4}(\xi) & =\left\{l_{3}(\xi) a_{11}^{(1)}+\left[l_{2}(\xi) l_{3}(\xi)-l_{1}(\xi) l_{4}(\xi)+l_{1}(\xi)\right] a_{21}^{(1)}\right\} /\left(\Delta^{\prime \prime} \Delta_{(1)}\right)
\end{aligned}
\end{aligned}
$$

Appendix $\mathrm{F}$

$p_{5}(\xi)=m_{1}(\xi)\left[f_{1}(\xi)-g_{1}(\xi)\right]\left(2+M_{T 1}^{2}-2 M_{L 1}^{2}\right), p_{6}(\xi)=2 \beta_{1} m_{3}(\xi)$

$p_{7}(\xi)=m_{2}(\xi)\left[f_{1}(\xi)-g_{1}(\xi)\right]\left(2+M_{T 1}^{2}-2 M_{L 1}^{2}\right), p_{8}(\xi)=2 \beta_{1} m_{4}(\xi)$

$p_{9}(\xi)=m_{1}(\xi)\left[f_{2}(\xi)-g_{2}(\xi)\right] \times 2 \alpha_{1}, p_{10}(\xi)=m_{1}(\xi)\left(M_{T_{1}}^{2}-2\right)$

$p_{11}(\xi)=m_{2}(\xi)\left[f_{2}(\xi)-g_{2}(\xi)\right] \times 2 \alpha_{1}, p_{12}(\xi)=m_{2}(\xi)\left(M_{T 1}^{2}-2\right)$

$p_{13}(\xi)=m_{1}^{\prime}(\xi)\left[f_{1}^{\prime}(\xi)-g_{1}^{\prime}(\xi)\right]\left(M_{T_{2}}^{2}-2\right), p_{14}(\xi)=-m_{1^{\prime}}(\xi) \times 2 \beta_{2}$

$p_{15}(\xi)=m_{2}^{\prime}(\xi)\left[f_{1}^{\prime}(\xi)-g_{1}^{\prime}(\xi)\right]\left(M_{T 2}^{2}-2\right), p_{16}(\xi)=-m_{2^{\prime}}(\xi) \times 2 \beta_{1}$

$p_{17}(\xi)=m_{1}^{\prime}(\xi)\left[f_{1}^{\prime}(\xi)-g_{1}^{\prime}(\xi)\right]\left(2+M_{T 2}^{2}-2 M_{L 2}^{2}\right), p_{18}(\xi)=2 \beta_{2} m_{3}^{\prime}(\xi)$

$p_{19}(\xi)=m_{2}^{\prime}(\xi)\left[f_{1}^{\prime}(\xi)-g_{1}^{\prime}(\xi)\right]\left(2+M_{T 2}^{2}-2 M_{L 2}^{2}\right), p_{20}(\xi)=2 \beta_{21} m_{4}^{\prime}(\xi)$

$p_{21}(\xi)=m_{1}^{\prime}(\xi)\left[f_{2}^{\prime}(\xi)-g_{2}^{\prime}(\xi)\right] \times 2 \alpha_{2}, p_{22}(\xi)=m_{1}^{\prime}(\xi)\left(M_{T 2}^{2}-2\right)$

$p_{23}(\xi)=m_{2}^{\prime}(\xi)\left[f_{2}^{\prime}(\xi)-g_{2}^{\prime}(\xi)\right] \times 2 \alpha_{21}, p_{24}(\xi)=m_{2}^{\prime}(\xi)\left(M_{T 2}^{2}-2\right)$

$m_{1}^{\prime}(\xi)=\left\{l_{2}(\xi) a_{22}^{(2)}+\left[1-l_{1}(\xi)\right] a_{12}^{(2)}\right\} /\left(\Delta^{\prime \prime} \Delta_{(2)}\right)$

$m_{2}^{\prime}(\xi)=\left\{l_{3}(\xi) a_{12}^{(2)}+\left[1-l_{4}(\xi)\right] a_{12}^{(2)}\right\} /\left(\Delta^{\prime \prime} \Delta_{(2)}\right)$

$m_{3}^{\prime}(\xi)=\left\{l_{2}(\xi) a_{21}^{(2)}+\left[1-l_{1}(\xi)\right] a_{11}^{(2)}\right\} /\left(\Delta^{\prime \prime} \Delta_{(2)}\right)$

$m_{4}^{\prime}(\xi)=\left\{l_{3}(\xi) a_{11}^{(2)}+\left[1-l_{4}(\xi)\right] a_{21}^{(2)}\right\} /\left(\Delta^{\prime \prime} \Delta_{(2)}\right)$

\section{References}

1) Erdogan, F. and Gupta, G., "The stress analysis of multilayered composites with a flaw," Int. J. Solids and Structures, Vol. 7, pp. 39-61(1971).

2) Sih, G. C. and Chen, E. P., "Normal and shear impact of layered composite with a crack: dynamic stress intensification," ASME J. Appl. Mech., Vol. 47, pp. 351-358(1980).

3) Yoffe, E. H., "The moving Griffith crack," Phil. Mag., Vol. 42, pp. 739-750(1951).

4) Sih, G. C. and Chen, E. P., "Moving cracks in layered compo sites,” Int. J. Engng. Sci., Vol. 20, pp. 1181-1192(1982).

5) Chen, S. H., Keer, L. M. and Achenbach, J. D., "Steady motion of a crack parallel to a bond-plane," Int. J. Engng. Sci., Vol. 18, pp. 225-238(1980).

6) Yau, W. F., “Axisymmetric slipless indentation of an infinite elastic cylinder," SIAM J. Appl. Math., Vol. 15, pp. 219227(1967).

7) Moriguchi, S., Udagawa, K. and Hitotsumatsu, S., "Tables of Mathematical Formulae III (in Japanese)," Iwanami-Shoten, Tokyo, (1987). 